\title{
Serum microRNA-1233 is a specific biomarker for diagnosing acute pulmonary embolism
}

Thorsten Kessler', Jeanette Erdmann ${ }^{2,3}$, Baiba Vilne', Petra Bruse², Volkhard Kurowski ${ }^{4}$, Patrick Diemert ${ }^{5}$, Heribert Schunkert ${ }^{1,6}$ and Hendrik B. Sager ${ }^{1 *}$

\begin{abstract}
Background: Circulating microRNAs (miRNAs) emerge as novel biomarkers in cardiovascular diseases. Diagnosing acute pulmonary embolism (PE) remains challenging due to a diverse clinical presentation and the lack of specific biomarkers. Here we evaluate serum miRNAs as potential biomarkers in acute PE.

Methods: We enrolled 30 patients with acute, CT (computed tomography)-angiographically confirmed central PE and collected serum samples on the day of emergency room admission (1st day) and from 22 of these patients 9 months thereafter. For comparison, we examined serum samples from patients with acute non ST-segment elevation myocardial infarction (NSTEMI, $n=30$ ) and healthy individuals $(n=12)$.

Results: We randomly selected 16 out of 30 PE patients and screened sera from the acute (1st day) and chronic stages ( 9 months) for 754 miRNAs using microarrays and found 37 miRNAs to be differentially regulated. Across all miRNAs, miRNA-1233 displayed the highest fold change (FC) from acute to chronic stage ( $\left.\log _{2} F C 11.5, p<0.004\right)$. We validated miRNA-1233 by real-time quantitative polymerase chain reaction (RT-qPCR). In acute PE (1 st day) we found elevated levels of miRNA-1233 in comparison to NSTEMI ( $\log _{2}$ FC 5.7, $\left.p<0.0001\right)$ and healthy controls $\left(\log _{2}\right.$ FC 7.7, $p<0.0001$ ). miRNA-1233 differentiated acute PE from NSTEMI patients and healthy individuals with 90 and $90 \%$ sensitivity, and 100 and $92 \%$ specificity [area under the curve (AUC) 0.95, $p<0.001$ and $0.91, p<0.001$ ], respectively.
\end{abstract}

Conclusions: This is the first report that identifies a miRNA that allows distinguishing acute PE from acute NSTEMI and healthy individuals with high specificity and sensitivity.

Keywords: Pulmonary embolism, Non ST-segment elevation myocardial infarction micro-RNA, Biomarker

\section{Background}

Acute pulmonary embolism (PE) is a common cardiovascular emergency with a high incidence of morbidity and mortality $[1,2]$. Together with deep vein thrombosis (DVT), PE is the third most frequent cardiovascular disease in western countries with an annual incidence of 100-300 per 100,000 individuals [3]. PE is difficult to diagnose and frequently missed due to a variable clinical presentation including dyspnea at rest or on exertion,

\footnotetext{
*Correspondence: hendrik.sager@tum.de

${ }^{1}$ Deutsches Herzzentrum München, Klinik für Herz- und

Kreislauferkrankungen, Technische Universität München, Lazarettstr. 36, 80636 Munich, Germany

Full list of author information is available at the end of the article
}

chest pain and syncope $[4,5]$. Patients with acute myocardial infarction (MI) also often present chest pain and shortness of breath, consequently acute $\mathrm{MI}$ is one of acute PE's most frequent differential diagnoses. The work-up for diagnosing PE can comprise biomarkers (such as the fibrin degradation product D-dimer), echocardiography, venous compression ultrasonography and radiologic imaging with computed tomography angiography, and ventilation-perfusion scintigraphy $[1,6]$. Although widely used in patients with suspected PE, D-dimer tests exhibit a high sensitivity, but fall short of being specific for diagnosing acute PE [7]. Hence, the introduction of novel biomarkers with a superior diagnostic accuracy would strongly facilitate diagnosing acute PE. 
MicroRNAs (miRNAs) are small ( 22 nucleotides), non-coding, single-stranded RNAs which can inhibit protein synthesis by negatively regulating gene expression via translational repression or mRNA degradation [8-10]. Often, miRNA expression is cell or tissue specific and their expression pattern can be altered upon initiation of pathologic processes. miRNAs can be detected in the circulation (serum or plasma) in a stable form [11] and thus represent attractive biomarkers for cardiovascular diseases [12-22].

In our study, we screened sera of patients with acute $\mathrm{PE}$ at different time points during the course of disease for differentially regulated miRNAs. We validated three selected miRNAs by qPCR and then tested their specificity and sensitivity for accurately diagnosing acute PE. This is the first report that identifies a profile of circulating miRNAs that allows distinguishing acute PE from acute non ST-segment elevation myocardial infarction (NSTEMI), acute DVT, chronic non-thromboembolic pulmonary hypertension $(\mathrm{PH})$ and healthy individuals with high specificity and sensitivity.

\section{Methods}

\section{Patients of the study group}

We enrolled patients presenting with acute symptom onset at the emergency department at the University Hospital of Schleswig-Holstein, Campus Lübeck, Germany who were diagnosed with central PE (location of the embolism in the pulmonary trunk and/or main pulmonary artery/ies) by CT-pulmonary angiography. Serum samples were taken at the emergency department on the admission day (1st day), as well as on the third and on the fifth day of the hospital stay. We recalled 22 patients $9 \pm 1.5$ months after the hospital stay and took a fourth serum sample.

\section{Patients of the control groups}

For comparison, we enrolled patients that presented at the emergency department with acute NSTEMI. Furthermore, we enrolled patients with acute DVT, patients with chronic non-thromboembolic $\mathrm{PH}$ and healthy individuals (colleagues in the lab without any current of past medical condition).

\section{Ethics, consent and permissions}

Patients gave informed consent to participate in this study as part of the Lübeck Registry of Structural Heart Disease [23], approved by the ethics committee of the University of Lübeck (No. 04-041).

\section{Consent to publish}

Patients gave informed consent for publishing results of this study as part of the Lübeck Registry of Structural Heart Disease [23].

\section{RNA isolation}

Blood samples were allowed to coagulate at room temperature and then immediately centrifuged at $1500 \mathrm{~g}$ for $15 \mathrm{~min}$. The supernatant was transferred to RNase/ DNase-free tubes and stored at $-80{ }^{\circ} \mathrm{C}$. RNA isolation was performed by using a miRNeasy Mini Kit (Qiagen, Hilden, Germany) with combining phenol/guanidinebased lysis and silicamembrane-based purification of RNA, according to the manufacturer's instructions. In brief, $300 \mu \mathrm{l}$ of serum were homogenized in $900 \mu \mathrm{l}$ of QIAzol lysis reagent (Qiagen, Hilden, Germany). After adding $240 \mu \mathrm{l}$ chloroform (Merck Millipore, Germany), the homogenate was separated by centrifugation. The upper (aqueous) phase was extracted and $100 \%$ ethanol (Merck Millipore, Germany) was added. Samples were then applied to miRNeasy Mini spin columns. RNA was eventually eluted in RNase-free water. To normalize for the miRNA content, we supplemented samples with 10 nM miRNA 39 from Caenorhabditis elegans (celmiR-39, Applied Biosystems, Foster City, CA, USA) as spiked-in control, after adding the QIAzol, as described previously [24, 25].

\section{Serum miRNA profiles}

We screened sera from PE patients for 754 miRNAs using TaqMan low-density miRNA microarrays (human miRNA A V2 and human miRNA B V3, Applied Biosystems, Foster City, CA, USA). Reverse transcription (RT) and pre-amplification steps were performed using the same volume of total RNA, according to the manufacturer's protocol. Real-time quantitative polymerase chain reaction (RT-qPCR) was performed using the $7900 \mathrm{HT}$ Fast Real-Time PCR System (Applied Biosystems, Foster City, CA, USA); results were expressed as Cts (cycle threshold, with the baseline set to 0.2 ).

\section{Validation of findings using miRNA RT-qPCR}

Based on the results from the screening experiments, we selected miRNAs for further RT-qPCR validation. After RNA extraction, and pre-amplification (carried out as described above), individual miRNA expression was determined using TaqMan microRNA Assays (Applied Biosystems, Foster City, CA, USA), according to manufacturer's instructions. We used the following TaqMan probes: hsa-miR-1233-002768, hsa-miR-27a\#-002445 and hsa-miR-134-001186 (Applied Biosystems, Foster City, CA, USA). In individual TaqMan microRNA Assays, cel-miRNA-39 was used for normalization.

\section{Computational methods miRNA screening}

miRNA array data analysis was performed using R/Bioconductor packages [26]. Raw threshold cycle $(\mathrm{Ct})$ values 
were first processed by replacing 'Undetermined' Ct values with $\mathrm{Ct}=50.0$ and thereafter filtered in a way that we excluded miRNAs where the median $\mathrm{Ct}$ was $\leq 35.0$ in at least one of the comparison groups ('acute' vs. 'chronic'). After filtering, raw $\mathrm{Ct}$ values were converted into relative quantities $(\mathrm{RQ})$ by using a formula $\mathrm{RQ}=\mathrm{E}^{\Delta \mathrm{Ct}}$, where PCR efficiency (E) was assumed to be $100 \%$ [27], reflected by a value of two for the base $E$ of the exponential function. Median normalization was used in order to obtain normalized relative quantities (NRQ) by dividing each $\mathrm{RQ}$ with the overall median of the corresponding sample ('acute' vs. 'chronic'). For two-way comparisons, the limma [28] t-statistic approach with Benjamini-Hochberg (BH; FDR) multiple testing correction [29] was used to select the differentially expressed miRNAs from the mediannormalized data. miRNAs were defined as differentially expressed if they had a $-1 \geq \log _{2} \mathrm{FC} \geq 1$ and $P$ value $\leq 0.15$ across the comparison groups ('acute' vs. 'chronic').

\section{miRNA validation}

Statistical analyses were carried out using GraphPad Prism software, version 6 (GraphPad Software, Inc.). Results are displayed as mean \pm standard error of mean (S.E.M.), if not noted otherwise. First, values were tested for outliers (ROUT method, Q = 1\%) and for Gaussian distribution (D'Agostino-Pearson omnibus normality test). For comparing more than two groups, an ordinary one-as applied to parametric data. For non-parametric data, a Kruskal-Wallis test was performed, followed by a Dunn's test for multiple comparisons. P values of $<0.05$ indicated statistical sway ANOVA test, followed by a Sidak's test for multiple comparisons, significance.

\section{ROC curve analyses}

All analyses were performed using $\mathrm{R}$ [30]. The diagnostic accuracy of individual miRNAs and their combinations was assessed using logistic regression and multinomial logistic regression analysis, respectively. The trade-offs between the specificity and sensitivity measures were then assessed using the receiver operating characteristic (ROC) curves (pROC package [31]). The area under the ROC curve (AUC) was used as summary measure to average the detection accuracy across the spectrum of test values. The AUC for each prediction was compared to random classifier ( $\mathrm{AUC}=0.5$ ) using the Wilcoxon signed rank test, as implemented in MKmisc http://crantastic.org/packages/MKmisc. The maximum value of the Youden's index $(J=$ Sensitivity + Specificity -1$)$ for each ROC curve was used for selecting the optimal cutoff point for the diagnostic tests. The positive likelihood ratio $(\mathrm{LR}+)$ was calculated as sensitivity/(1 - specificity), the negative likelihood ratio (LR-) as $(1-$ Sensitivity)/ Specificity.

\section{Results}

\section{Patients and serum sampling}

We enrolled 30 patients (age $62 \pm 14$, mean \pm SD, $57 \%$ male, all Caucasians) who presented with acute symptom onset at the emergency department and were here diagnosed with central PE (location of the embolism in the pulmonary trunk and/or main pulmonary artery/ ies) by computed tomography (CT)-pulmonary angiography (Table 1 ). The majority of patients presented with shortness of breath $(81 \%)$ and/or chest pain (33\%). In most patients the cause of PE remained idiopathic. The time course of biomarkers D-dimers, high sensitive troponin $\mathrm{T}$ and $\mathrm{n}$-terminal ( $\mathrm{nt}$ ) pro brain natriuretic peptide (BNP) is displayed in Fig. 1. Serum samples were taken at the emergency department on the admission day (1st day), as well as on the third and fifth day of the hospital stay. We re-called 22 (50\% male patients) of these 30 patients $9 \pm 1.5$ months (mean \pm SD) after the hospital stay and collected a fourth serum sample ( 9 months). For comparison, we enrolled 30 age- and gender-matched

\section{Table 1 Patients' characteristics}

\begin{tabular}{|c|c|}
\hline$n$ & 30 \\
\hline Age (mean $\pm S D)$ & $62 \pm 14$ \\
\hline Male & $17 / 30(57 \%)$ \\
\hline $\begin{array}{l}\text { CTA confirmed central PE (pulmonary trunk, main pulmo- } \\
\text { nary arteries) }\end{array}$ & $100 \%$ \\
\hline \multicolumn{2}{|l|}{ Risk stratefication (PE-related early mortality rate) } \\
\hline High $(>15 \%)$ & $4 / 30(13 \%)$ \\
\hline Intermediate (3-15 \%) & $15 / 30(50 \%)$ \\
\hline $\operatorname{Low}(<1 \%)$ & $11 / 30(37 \%)$ \\
\hline \multicolumn{2}{|l|}{ Symptoms } \\
\hline Dyspnoea & $24 / 30(81 \%)$ \\
\hline Chest pain & $10 / 30(33 \%)$ \\
\hline Cough & $3 / 30(10 \%)$ \\
\hline Syncope & $3 / 30(10 \%)$ \\
\hline Haemoptysis & $2 / 30(7 \%)$ \\
\hline Collaps with subsequent CPR & $4 / 30(13 \%)$ \\
\hline Idiopathic DVT/PE & $13 / 30(43 \%)$ \\
\hline \multicolumn{2}{|l|}{ Secondary DVT/PE } \\
\hline Malignancy & 4/30 (13\%) \\
\hline Post surgery & $4 / 30(13 \%)$ \\
\hline Immobilisation & $2 / 30(7 \%)$ \\
\hline Thrombophilia & 1/30 (3 \%) \\
\hline Previous DVT/PE & $2 / 30(7 \%)$ \\
\hline Oral contraceptive therapy & 2/30 (7 \%) \\
\hline Obesity (BMI $\left.\geq 30 \mathrm{~kg} / \mathrm{m}^{2}\right)$ & $12 / 30(40 \%)$ \\
\hline US confirmed DVT & $25 / 30(83 \%)$ \\
\hline
\end{tabular}

Characteristics of acute pulmonary embolism (PE) patients at the time of the emergency room presentation (1st day)

CTA computed tomography (pulmonary) angiography, CPR cardiopulmonary resuscitation, DVT deep vein thrombosis, BMI body mass index, US ultrasound, $S D$ standard deviation 


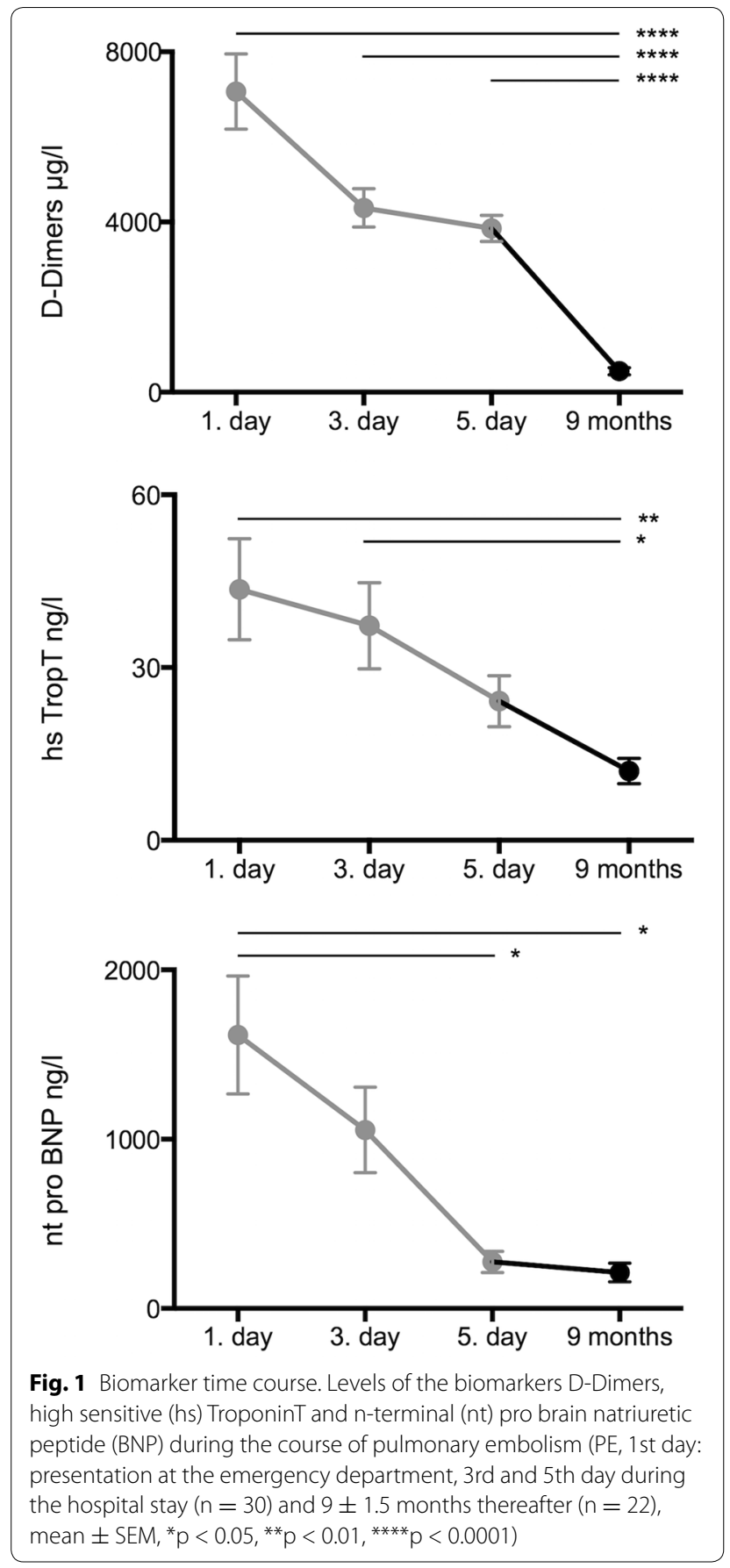

patients that presented at the emergency department with acute non ST-segment elevation myocardial infarction (NSTEMI, mean age \pm SD: $64 \pm 13,57 \%$ males, Table 2). Furthermore, we enrolled six age-matched patients with acute DVT (mean age \pm SD: $66 \pm 10,67 \%$ males) confirmed by compression ultrasonography without any clinical or apparative signs of concomitant PE (no dyspnea, no chest pain, normal ECG, normal right
Table 2 Patients' characteristics

\begin{tabular}{|c|c|c|c|}
\hline & Acute PE & Acute NSTEMI & $\mathbf{p}$ \\
\hline Age $(y)$ & $62 \pm 14$ & $64 \pm 13$ & 0.57 \\
\hline Male gender (\%) & 57 & 57 & 1.00 \\
\hline Onset to sampling < 24 h (\%) & 63 & 70 & 0.58 \\
\hline $\operatorname{BMI}\left(\mathrm{kg} / \mathrm{m}^{2}\right)$ & $30.6 \pm 7.7$ & $27.9 \pm 9.3$ & 0.23 \\
\hline LV-EF (\%) & $60 \pm 2$ & $57 \pm 7$ & 0.03 \\
\hline \multicolumn{4}{|l|}{ CV risk factors } \\
\hline Hypertension (\%) & 63 & 81 & 0.15 \\
\hline Hyperlipidemia (\%) & 17 & 41 & 0.04 \\
\hline Smoking (\%) & 10 & 52 & $<0.001$ \\
\hline Diabetes mellitus (\%) & 20 & 22 & 0.75 \\
\hline Positive family history (\%) & 3 & 33 & $<0.01$ \\
\hline \multicolumn{4}{|l|}{ CVhistory } \\
\hline Non-obstructive CAD (\%) & 17 & 3 & 0.09 \\
\hline Obstructive CAD (\%) & 0 & 6 & 0.15 \\
\hline Previous PCI/CABG (\%) & 0 & 6 & 0.15 \\
\hline Previous MI (\%) & 0 & 6 & 0.15 \\
\hline Resuscitation (\%) & 0 & 0 & 1.00 \\
\hline Atrial fibrillation (\%) & 0 & 3 & 0.31 \\
\hline Previous stroke (\%) & 3 & 3 & 1.00 \\
\hline
\end{tabular}

Patients' characteristics of the acute pulmonary embolism (PE) and acute non ST-segment elevation myocardial infarction (NSTEMI) groups

$B M I$ body mass index, $L V-E F$ left ventricular ejection fraction, $C V$ cardiovascular, $C A D$ coronary artery disease, $P C l$ percutaneous coronary intervention, $C A B G$ coronary artery bypass grafting, $M I$ myocardial infarction

heart echocardiography, normal blood gas analysis), 15 age-matched patients with chronic non-thromboembolic pulmonary hypertension $(\mathrm{PH}$, mean age $\pm \mathrm{SD}: 65 \pm 13$, $47 \%$ males), and 12 healthy individuals (mean age \pm SD: $31 \pm 6,50 \%$ males).

\section{Serum miRNA screening and validation}

We first randomly selected 16 out of $30 \mathrm{PE}$ patients and screened their sera from the acute phase (1st day) and from the chronic stage ( 9 months) for 754 miRNAs using TaqMan human miRNA arrays. We found 37 miRNAs to be differentially regulated with a nominally significant $P$ value $(<0.05,1$ st day vs. 9 months, Fig. 2 a). Across all screened miRNAs, miRNA-1233 displayed the highest fold change at the acute (1st day) in comparison to the chronic stage (9 months) $\left(\log _{2} \mathrm{FC} 11.5, P<0.004\right)$. We then validated our array findings on all enrolled $\mathrm{PE}$ patients from all time points by TaqMan-based miRNA real-time quantitative polymerase chain reaction (RTqPCR). We confirmed that miRNA-1233 displayed highest serum levels on the first day, its levels then decreased on the third and fifth day and were lowest at the chronic stage of the disease (FC 3.7 on 1st day vs. 9 months, $\mathrm{p}<0.01$, Fig. 2b). Our findings show that miRNA-1233 was up-regulated in the serum of acute PE patients, 
a

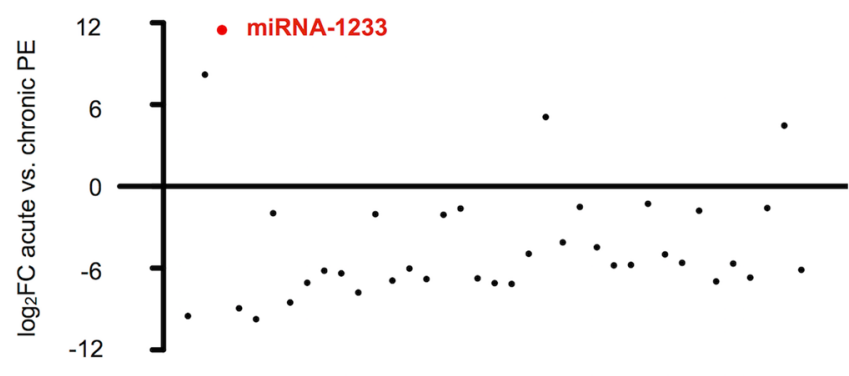

b

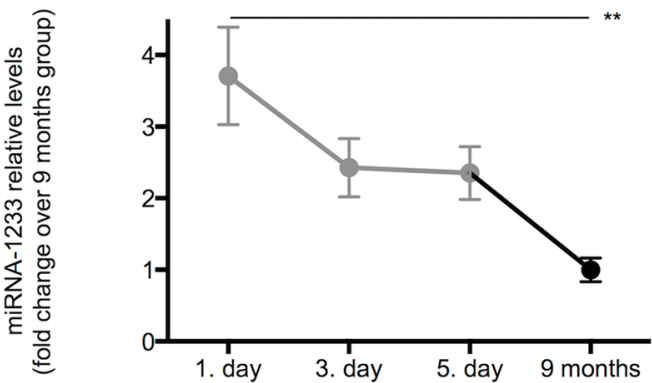

C

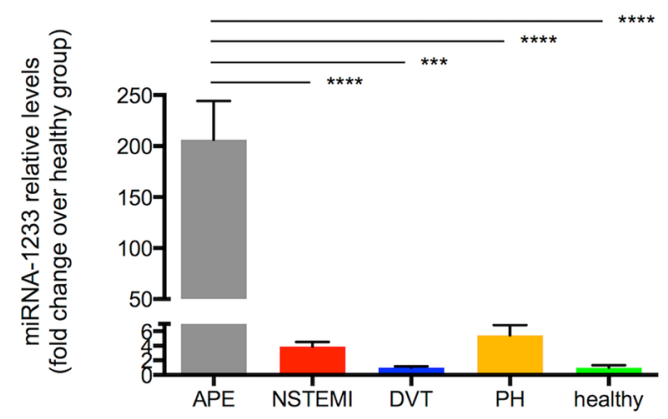

d

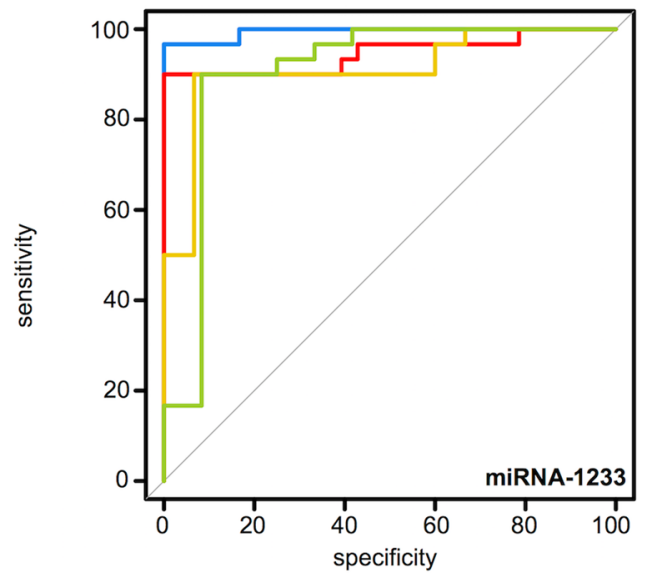

$\square$ acute PE vs. NSTEMI $\square$ acute PE vs. PH

acute PE vs. DVT $\square$ acute PE vs. healthy

Fig. 2 miRNA-1233. a microRNA (miRNA) array screening. We randomly selected 16 out of 30 pulmonary embolism (PE) patients and screened their sera from the acute (1st day) and chronic stage (9 months) for 754 miRNAs. The 37 most differentially expressed miRNAs (nominal P value $\leq 0.05$ ) were displayed. miRNA-1233 (red dot) displayed the highest fold change on the 1st day ( $\log _{2}$ FC 11.5 on 1 st day vs. 9 months, $p<0.004$ ) and was consequently subjected to real-time quantitative polymerase chain reaction (RT-qPCR) validation. b miRNA RT-qPCR validation. Serum miRNA-1233 levels during the time course of acute PE (1 st day: presentation at the emergency department, 3rd and 5th day during the hospital stay ( $n=30$ ) and $9 \pm 1.5$ months thereafter $(n=22)$. Values represent relative levels with the "9 months" group set as 1 (mean $\pm S E M$, ${ }^{* *} p<0.01$ ). c Serum miRNA-1233 levels from acute pulmonary embolism patients (APE, 1st day, $n=30$ ) in comparison to patients with acute non ST-segment elevation myocardial infarction (NSTEMI, $n=30$ ), acute deep vein thrombosis without concomitant PE (DVT, $n=6)$, chronic non-thromboembolic pulmonary hypertension $(\mathrm{PH}, \mathrm{n}=15)$ and healthy individuals $(n=12)$. Values represent relative levels with the "healthy" group set as 1 (mean \pm SEM, $\left.{ }^{* * *} p<0.001,{ }^{* * * *} p<0.0001\right)$. d Receiver operating characteristic (ROC) curve analysis for miRNA-1233 to discriminate acute PE (1st day) from acute NSTEMI (red line, area under the curve (AUC) $0.95, \mathrm{p}<0.001$, sensitivity $90 \%$ and specificity $100 \%$ ), acute DVT (blue line, AUC 0.99, p $<0.001$, sensitivity $97 \%$ and specificity $100 \%$ ), chronic PH (orange line, AUC 0.91, p<0.001, sensitivity $90 \%$ and specificity $93 \%$ ) and healthy individuals (green line, AUC $0.91, p<0.001$, sensitivity $90 \%$ and specificity $92 \%)$

indicating that it could represent a marker for diagnosing acute PE.

\section{Sensitivity and specificity of miRNA-1233 for diagnosing acute $\mathrm{PE}$}

We then tested miRNA-1233 specificity and sensitivity for diagnosing acute PE and compared miRNA1233 serum levels from acute PE patients to levels from patients with acute NSTEMI, acute DVT, chronic non-thromboembolic $\mathrm{PH}$ and healthy individuals by
'TaqMan-based miRNA RT-qPCR. Patients' characteristics are shown in Table 2. We found significantly higher levels of miRNA-1233 in the serum of acute PE patients in comparison to all other groups (PE 1st day FC 206.3, NSTEMI FC 3.9, DVT FC 1, PH FC 5.4 vs. healthy, Fig. 2c). Receiver operating characteristic (ROC) analysis revealed that miRNA-1233 differentiated acute PE (1st day) from acute NSTEMI, DVT, PH patients and healthy controls with high sensitivity (90, 97, 90, and $90 \%$, respectively) and specificity $(100,100,93$, and $92 \%$, 
respectively, Fig. 2c). Detailed ROC statistics are shown in Table 3. Taken together, miRNA-1233 distinguished acute PE (1st day) from acute NSTEMI, its most frequent differential diagnosis, with high specificity and sensitivity.

\section{miRNA profile/signature}

We further identified two additional miRNAs, miRNA27a and miRNA-134, which exhibited highest serum levels in PE patients on the first day and lowest levels at 9 months (FC 3.6, 4.1 on 1 st day vs. 9 months, $\mathrm{p}<0.05$, $\mathrm{p}<0.01$, respectively, Fig. 3a). Similar to miRNA-1233, we found significantly higher levels of miRNAs $-27 \mathrm{a}$ and 134 in the serum of acute PE patients in comparison to all other groups (PE 1st day FC 34.1, 14.8; NSTEMI FC 2.3, 2.5; DVT FC 1.4, 0.3; PH FC 7.5, 3.3 vs. healthy, respectively, Fig. 3b). ROC analysis revealed that miRNA-27a differentiated acute PE (1st day) from NSTEMI, DVT, PH patients and healthy controls with 63, 73, 67, and $67 \%$ sensitivity, and $89,100,80$, and $92 \%$ specificity, respectively (Fig. 3c). Accordingly, miRNA-134 differentiated acute PE (1st day) from NSTEMI, DVT, PH patients and healthy controls with $83,80,70$, and $83 \%$ sensitivity, and 64, 100, 73, and $83 \%$ specificity, respectively (Fig. 3c). Detailed ROC statistics are shown in Table 3. We then tested, whether or not a combination of miRNAs (serum miRNA profile/signature) improved diagnostic sensitivity

Table 3 Receiver operating characteristic curves

\begin{tabular}{|c|c|c|c|c|c|c|c|c|c|c|}
\hline & AUC & $95 \% \mathrm{Cl}$ & $\mathbf{p}$ & Cut-off & Sensitivity (\%) & $95 \% \mathrm{Cl}$ & Specificity (\%) & $95 \% \mathrm{Cl}$ & $\mathrm{LR}+$ & LR- \\
\hline \multicolumn{11}{|l|}{ Acute PEvs. healthy } \\
\hline miR-27a & 0.79 & $0.65-0.93$ & $<0.01$ & 0.63 & 67 & $50-83$ & 92 & $75-100$ & 8 & 0.4 \\
\hline miR-134 & 0.84 & $0.71-0.96$ & $<0.001$ & 0.51 & 83 & 70-97 & 83 & $58-100$ & 5 & 0.2 \\
\hline miR-1233 & 0.91 & $0.82-0.99$ & $<0.001$ & 0.53 & 90 & $77-100$ & 92 & $75-100$ & 10.8 & 0.1 \\
\hline miR-27a + miR-134 & 0.88 & $0.78-0.98$ & $<0.001$ & 0.47 & 90 & $77-100$ & 83 & $58-100$ & 5.4 & 0.1 \\
\hline miR-27a + miR-1233 & 0.89 & $0.79-0.99$ & $<0.001$ & 0.48 & 97 & $90-100$ & 83 & $58-100$ & 5.8 & 0 \\
\hline miR-134 + miR-1233 & 0.88 & $0.77-0.98$ & $<0.001$ & 0.40 & 93 & $83-100$ & 75 & $50-100$ & 3.7 & 0 \\
\hline miR-27a + miR-134 + miR-1233 & 0.90 & $0.81-0.99$ & $<0.001$ & 0.50 & 90 & $80-100$ & 83 & $58-100$ & 5.4 & 0.1 \\
\hline \multicolumn{11}{|l|}{ Acute PE vs. NSTEMI } \\
\hline miR-27a & 0.78 & $0.66-0.90$ & $<0.001$ & 0.46 & 63 & $43-80$ & 89 & $79-100$ & 5.9 & 0.4 \\
\hline miR-134 & 0.78 & $0.66-0.90$ & $<0.001$ & 0.74 & 83 & $70-97$ & 64 & $46-82$ & 2.3 & 0.3 \\
\hline miR-1233 & 0.95 & $0.89-1.00$ & $<0.001$ & 0.55 & 90 & $80-100$ & 100 & $100-100$ & $\infty$ & 0.1 \\
\hline miR-27a + miR-134 & 0.80 & $0.68-0.91$ & $<0.001$ & 0.34 & 87 & $73-97$ & 61 & $43-79$ & 2.2 & 0.2 \\
\hline miR-27a + miR-1233 & 0.96 & $0.91-1.00$ & $<0.001$ & 0.69 & 90 & $77-100$ & 100 & $100-100$ & $\infty$ & 0.1 \\
\hline miR-134 + miR-1233 & 0.95 & $0.89-1.00$ & $<0.001$ & 0.54 & 90 & $77-100$ & 100 & $100-100$ & $\infty$ & 0.1 \\
\hline miR-27a + miR-134 + miR-1233 & 0.97 & $0.93-1.00$ & $<0.001$ & 0.71 & 90 & $77-100$ & 100 & $100-100$ & $\infty$ & 0.1 \\
\hline \multicolumn{11}{|l|}{ AcutePEvs.DVT } \\
\hline $\operatorname{miR}-27 a$ & 0.83 & $0.67-0.98$ & $<0.05$ & 0.79 & 73 & $57-90$ & 100 & $100-100$ & $\infty$ & 0.3 \\
\hline miR-134 & 0.84 & $0.72-0.99$ & $<0.01$ & 0.74 & 80 & $67-93$ & 100 & $100-100$ & $\infty$ & 0.2 \\
\hline miR-1233 & 0.99 & $0.97-1.0$ & $<0.001$ & 0.72 & 97 & $90-100$ & 100 & $100-100$ & $\infty$ & 0 \\
\hline miR-27a + miR-134 & 0.92 & $0.83-1.0$ & $<0.001$ & 0.63 & 87 & $73-97$ & 100 & $100-100$ & $\infty$ & 0.1 \\
\hline miR-27a + miR-1233 & 0.99 & $0.97-1.0$ & $<0.001$ & 0.72 & 97 & $90-100$ & 100 & $100-100$ & $\infty$ & 0 \\
\hline miR-134 + miR-1233 & 0.99 & $0.97-1.0$ & $<0.001$ & 0.72 & 97 & $90-100$ & 100 & $90-100$ & $\infty$ & 0 \\
\hline miR-27a + miR-134 + miR-1233 & 0.99 & $0.97-1.0$ & $<0.01$ & 0.72 & 97 & $90-100$ & 100 & $100-100$ & $\infty$ & 0 \\
\hline \multicolumn{11}{|l|}{ Acute PE vs. PHT } \\
\hline miR-27a & 0.66 & $0.50-0.82$ & 0.085 & 0.66 & 67 & $50-83$ & 80 & $60-100$ & 3.3 & 0.4 \\
\hline miR-134 & 0.67 & $0.51-0.83$ & 0.062 & 0.61 & 70 & $53-87$ & 73 & $47-93$ & 2.6 & 0.4 \\
\hline miR-1233 & 0.91 & $0.83-0.99$ & $<0.001$ & 0.43 & 90 & $80-100$ & 93 & $80-100$ & 13.5 & 0.1 \\
\hline miR-27a + miR-134 & 0.66 & $0.50-0.82$ & 0.085 & 0.59 & 70 & $53-87$ & 73 & $47-93$ & 2.6 & 0.4 \\
\hline miR-27a + miR-1233 & 0.92 & $0.84-1.0$ & $<0.001$ & 0.42 & 90 & $77-100$ & 93 & $80-100$ & 13.5 & 0.1 \\
\hline miR-134 + miR-1233 & 0.91 & $0.83-0.99$ & $<0.001$ & 0.42 & 90 & $77-100$ & 93 & $80-100$ & 13.5 & 0.1 \\
\hline miR-27a + miR-134 + miR-1233 & 0.90 & $0.81-0.99$ & $<0.001$ & 0.46 & 90 & $80-100$ & 93 & $80-100$ & 13.5 & 0.1 \\
\hline
\end{tabular}

Receiver operating characteristic (ROC) curves. Cut-off is defined as the Youden's index which represents the point on the curve furthest away from the 45 degree line AUC area under the curve, $C I$ confidence interval, $L R+$ positive likelihood ratio, $L R$ - negative likelihood ratio, miR microRNA, $P E$ pulmonary embolism, NSTEMI acute non-ST segment myocardial infarction, DVT acute deep vein thrombosis without concomitant $\mathrm{PE}, \mathrm{PH}$ chronic non-thromboembolic pulmonary hypertension 

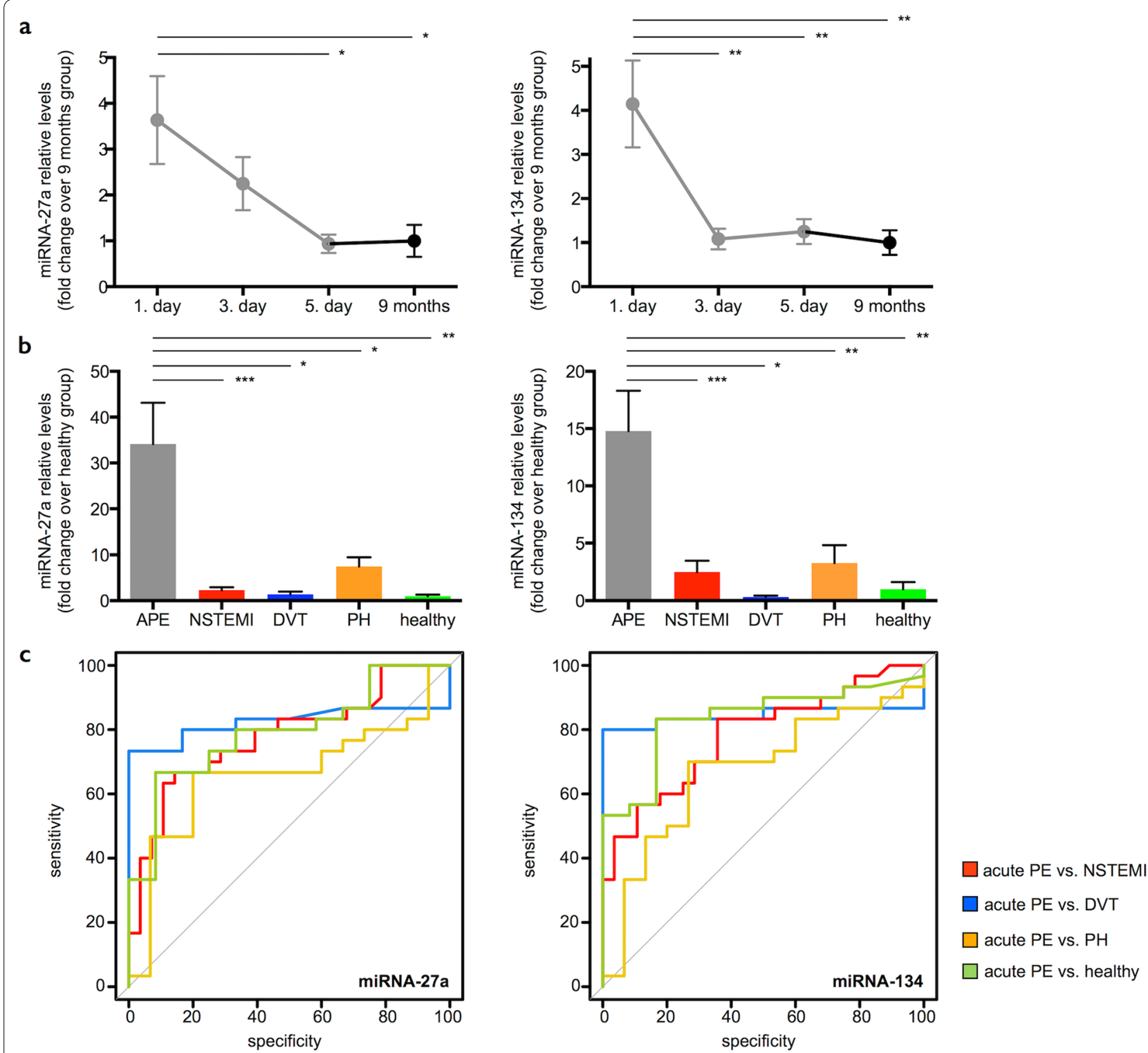

Fig. 3 miRNA-27a and 134. a Serum miRNA-27a and 134 levels during the time course of acute pulmonary embolism (APE, 1st day: presentation at the emergency department, 3rd and 5th day during the hospital stay $(n=30)$ and $9 \pm 1.5$ months thereafter $(n=22)$. Values represent relative levels with the pulmonary embolism (PE) "9 months" group set as 1 (mean \pm SEM, ${ }^{*} p<0.05,{ }^{* *} p<0.01$ ). b Serum miRNA-27a and 134 levels from acute PE patients (APE, 1st day, $n=30$ ) in comparison to patients with acute non ST-segment elevation myocardial infarction $(N S T E M I, n=30)$, acute deep vein thrombosis without concomitant $P E(D V T, n=6)$, chronic non-thromboembolic pulmonary hypertension $(P H, n=15)$ and healthy individuals $(n=12)$. Values represent relative levels with the "healthy" group set as 1 (mean $\left.\pm S E M,{ }^{*} p<0.05,{ }^{* *} p<0.01,{ }^{* * *} p<0.001\right)$. c ROC (Receiver operating characteristic) curve analysis for miRNA-27a and 134 to discriminate acute PE (1st day) from acute NSTEMI (red line, area under the curve (AUC) $0.78,0.78, p<0.001,<0.001$, respectively), acute DVT (blue line, AUC 0.83, 0.84, $p<0.05,<0.01$, respectively), chronic PH (orange line, AUC 0.66, $0.67, p=0.09,0.06$, respectively) and healthy individuals (green line, AUC $0.79,0.84, p<0.01,<0.001$, respectively)

and specificity. Neither combining miRNA-1233 with either miRNA-27a or miRNA-134 (Fig. 4a; Table 3), nor combining all three miRNAs improved sensitivity and specificity for accurately differentiating acute PE from healthy individuals and acute NSTEMI patients (Fig. 4b; Table 3 ). We then directly compared all three miRNA and found that miRNA-1233 was always superior to miRNAs-27a and miRNA-134 for accurately distinguishing acute PE from acute NSTEMI, acute DVT and PH patients and from healthy individuals (Fig. 4c; Table 3). In summary, miRNA-1233 alone yielded a superior capability of distinguishing acute PE from healthy individuals 

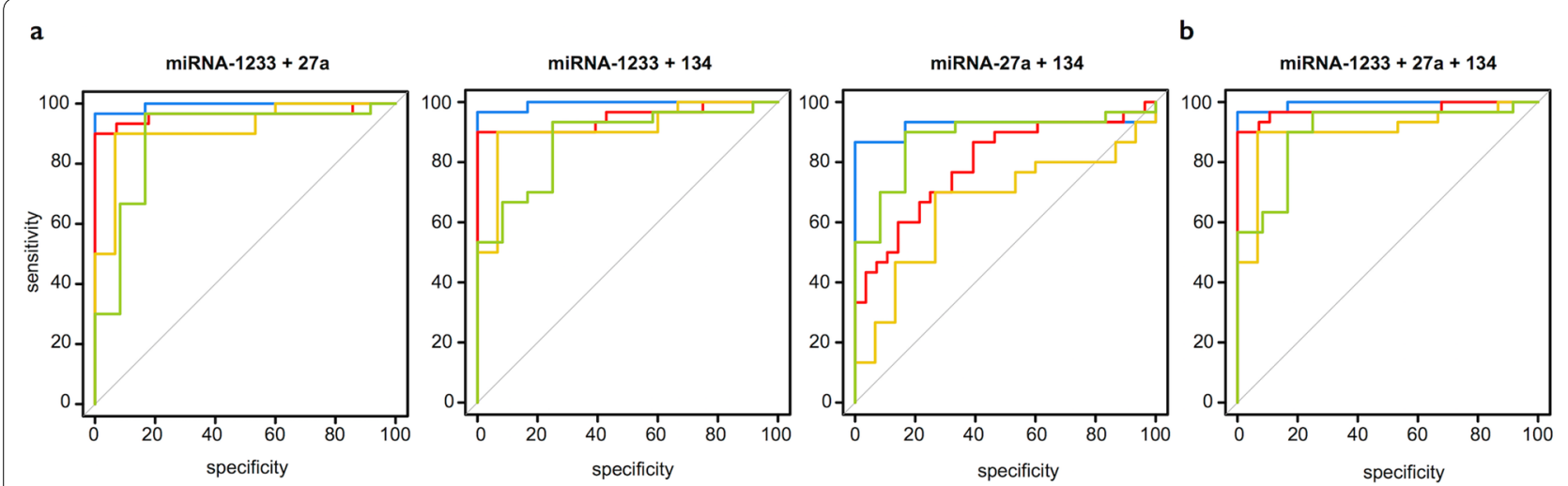

$\square$ acute PE vs. NSTEMI $\square$ acute PE vs. healthy

$\square$ acute PE vs. DVT $\square$ acute PE vs. PH

C
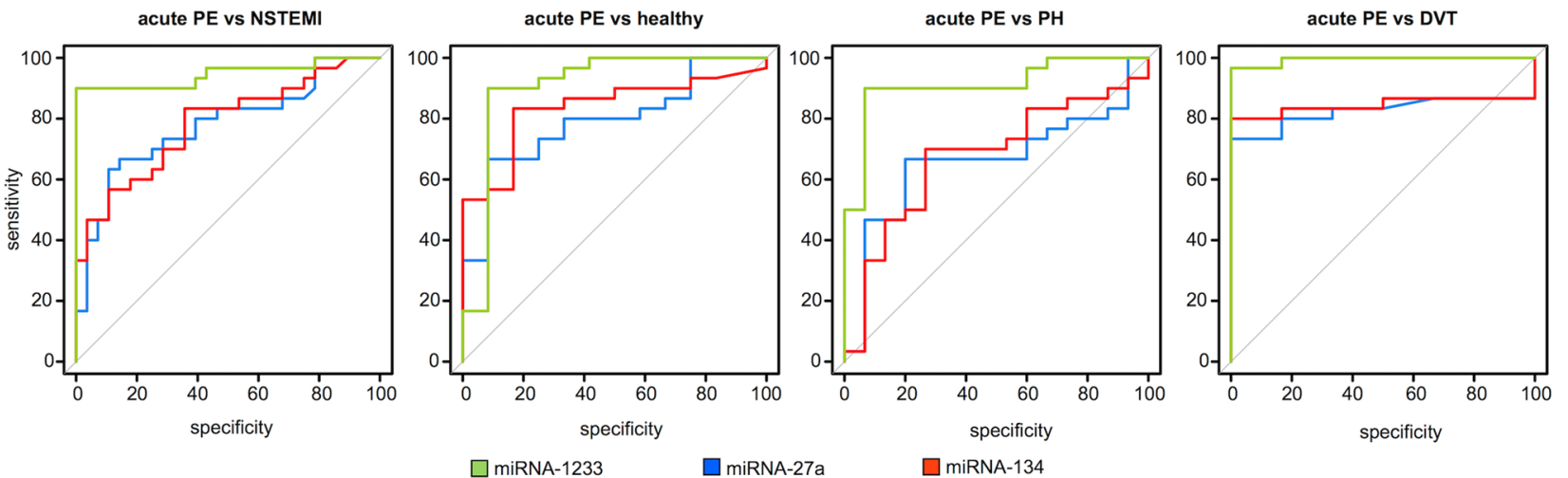

Fig. 4 Receiver operating characteristic curve analysis. ROC (Receiver operating characteristic) curve analysis for a combining two microRNAs (miRNA) or b all three miRNAs to discriminate acute pulmonary embolism (PE, 1st day) from acute non-ST segment myocardial infarction (NSTEMI, red line), acute deep vein thrombosis without concomitant PE (DVT, blue line), chronic non-thromboembolic pulmonary hypertension (PH, orange line) and healthy individuals (green line). c ROC curve analysis for comparing miRNAs (green line: miRNA-1233, red line: miRNA-134, blue line: miRNA27a) to discriminate acute PE (1st day) from acute NSTEMI, acute DVT without concomitant PE, chronic non-thromboembolic PH and healthy individuals

and the described diseases in comparison to miRNA-27a and miRNA-134 (Table 3). A signature, comprising miRNAs-1233, 27a and 134 could not further increase sensitivity and specificity, and was consequently not superior to miRNA-1233 alone.

\section{Discussion}

A timely diagnosis of $\mathrm{PE}$ remains to be a challenging clinical problem [3, 32]. In fact, recent studies have revealed that up to one third of all PE cases are missed at the emergency department [33]. Here, we screened sera of PE patients for more than 750 miRNA by using miRNA arrays and found significantly elevated levels of miRNA-1233 in patients with acute PE (vs. chronic PE). We validated these array findings by individual TaqMan qPCR and found-as compared to acute PE patientsmarkedly lower miRNA-1233 levels in patients with NSTEMI, DVT, PH, as well as in healthy individuals.
Importantly, miRNA-1233 distinguished acute PE from acute NSTEMI, its most frequent differential diagnosis, with high specificity and sensitivity.

Pulmonary embolism is a frequent emergency affecting 100-300/100,000 individuals per year in industrialized nations [3]. Like acute MI patients, patients with acute PE present symptoms like chest pain and shortness of breath. Consequently, acute PE and acute MI are among the most frequent differential diagnosis for shortness of breath and chest pain [3, 4], and distinguishing these diseases can be difficult due to a similar clinical presentation. However, distinguishing PE from MI early on is crucial because these different pathologies require distinct treatments, which, when applied in a timely fashion, significantly enhance patient survival [34-37]. PE treatment is mainly conservative and focuses on anticoagulation, while in $\mathrm{MI}$ an invasive re-vascularization therapy is often pursued [38]. 
To date, there are no specific biomarkers for an early accurate detection of acute PE. High sensitivity troponins and D-dimers are often used for diagnosing MI or PE, respectively. However, these markers do not perfectly discriminate the two conditions. Specifically, elevated D-dimer levels can also be found in MI patients [39], as well as in a number of other conditions [1]. Vice versa, elevated troponin levels can also be detected in acute PE, especially if the right heart is affected (acute cor pulmonale) [40].

Rapidly and accurately diagnosing acute PE would be an extremely helpful tool, especially at emergency departments. Ideally, miRNA-1233 could identify acute PE patients early as a bedside test so that correct treatment could be initiated timely, consequently reducing mortality and morbidity. As of now, most laboratories use RT-qPCR-based methods for detecting serum miRNAs. RT-qPCR is very sensitive on one hand, but also difficult to standardize on the other. To date, a house keeping miRNA to normalize miRNA content to is lacking. The current practice of supplementing external controls (for instance spiking of miRNAs for normalization in PCR-based measurements) might not be sufficient enough to provide an accurate bedside measurement of circulating miRNAs. For the clinical routine, well-defined cutoff values and reliable measurements are most crucial and would be needed.

To date, there is one other study that also evaluated serum miRNAs for diagnosing PE [18]. We reproduced the finding of Xiao et al. that miRNA-134 is up-regulated in acute PE patients making miRNA-134 an even more promising marker, since our findings are the first replication on an independent cohort. However, the study did not report on miRNA-1233 or on miRNA-27a, and nor did it show miRNA-134 specificity to distinguish PE from other relevant differential diagnosis like NSTEMI.

Interpretations of our study results are limited mainly due to (1) a rather small sample size $(\mathrm{n}=30$ in each the PE and NSTEMI group) and (2) a highly selected, very morbid group of PE patients with a high thrombus burden. Our findings need to be replicated on a larger cohort of patients and also on less morbid PE patients with peripheral rather than central emboli.

\section{Conclusions}

Circulating miRNA-1233 allows distinguishing acute PE from acute NSTEMI and healthy individuals with high specificity and sensitivity, and consequently appears to be a promising marker for accurately diagnosing acute PE.

\section{Authors' contributions}

TK and HBS designed and managed the study, designed and performed experiments, collected and analyzed the data, and wrote the manuscript which was edited and approved by all co-authors; BV, PB, and SH performed experiments, collected, analyzed, and discussed data. JE, VK, PD, and HS conceived experiments and discussed strategy and results. All authors read and approved the final manuscript.

\section{Author details}

${ }^{1}$ Deutsches Herzzentrum München, Klinik für Herz- und Kreislauferkrankungen, Technische Universität München, Lazarettstr. 36, 80636 Munich, Germany. ${ }^{2}$ Institut für Integrative und Experimentelle Genomik, Universität zu Lübeck, Lübeck, Germany. ${ }^{3}$ Deutsches Zentrum für Herz-Kreislauf-Forschung (DZHK) e.V., partner site Hamburg/Lübeck/Kiel, Lübeck, Germany. ${ }^{4}$ Innere Medizin, DRK-Krankenhaus Mölln-Ratzeburg, Ratzeburg, Germany. ${ }^{5}$ Medizinische Klinik II, Westküstenklinikum, Heide, Germany. ${ }^{6}$ Deutsches Zentrum für Herz-Kreislauf-Forschung (DZHK) e.V., partner site Munich Heart Alliance (MHA), Munich, Germany.

\section{Acknowledgements}

This work was funded in part by Grants from The Section Of Medicine, University of Lübeck, Germany (E30-2012). Hendrik B. Sager was supported by the Deutsche Forschungsgemeinschaft (SA1668/2-1). Thorsten Kessler was supported by the Deutsches Zentrum für Herz-Kreislauf-Forschung (DZHK). The study was also supported by the German Federal Ministry of Education and Research (BMBF) in the context of the e:Med program (e:AtheroSysMed) and the FP7 European Union project CVgenes@target (261123). Further Grants were received by the Fondation Leducq (CADgenomics: Understanding CAD Genes, 12CVD02) and the Deutsche Forschungsgemeinschaft as part of the Sonderforschungsbereich CRC 1123 (B2). This work was supported by the German Research Foundation (DFG) and the Technische Universität München within the funding programme Open Access Publishing.

\section{Competing interests}

The authors declare that they have no competing interests.

Received: 4 March 2016 Accepted: 28 April 2016

Published online: 05 May 2016

\section{References}

1. Konstantinides SV, Torbicki A, Agnelli G, Danchin N, Fitzmaurice D, Galiè $\mathrm{N}$, et al. 2014 ESC guidelines on the diagnosis and management of acute pulmonary embolism. Eur Heart J. 2014;35:3033-69.

2. Jaff MR, McMurtry MS, Archer SL, Cushman M, Goldenberg N, Goldhaber $\mathrm{SZ}$, et al. Management of massive and submassive pulmonary embolism, iliofemoral deep vein thrombosis, and chronic thromboembolic pulmonary hypertension: a scientific statement from the American Heart Association. Circulation. 2011;123:1788-830.

3. Douma RA, Kamphuisen PW, Buller HR. Acute pulmonary embolism. Part 1: epidemiology and diagnosis. Nat Rev Cardiol. 2010;7:585-96.

4. Agnelli G, Becattini C. Acute pulmonary embolism. N Engl J Med. 2010;363:266-74.

5. Huisman MV, Klok FA. How I diagnose acute pulmonary embolism. Blood. 2013;121:4443-8.

6. Moores LK, King CS, Holley AB. Current approach to the diagnosis of acute nonmassive pulmonary embolism. Chest. 2011;140:509-18.

7. Stein PD, Hull RD, Patel KC, Olson RE, Ghali WA, Brant R, et al. D-dimer for the exclusion of acute venous thrombosis and pulmonary embolism: a systematic review. Ann Intern Med. 2004;140:589-602.

8. Booton R, Lindsay MA. Emerging role of MicroRNAs and long noncoding RNAs in respiratory disease. Chest. 2014;146:193-204.

9. Pritchard CC, Cheng HH, Tewari M. MicroRNA profiling: approaches and considerations. Nat Rev Genet. 2012;13:358-69.

10. van Rooij E, Olson EN. MicroRNA therapeutics for cardiovascular disease: opportunities and obstacles. Nat Rev Drug Discov. 2012;11:860-72.

11. Xu J, Zhao J, Evan G, Xiao C, Cheng Y, Xiao J. Circulating microRNAs: novel biomarkers for cardiovascular diseases. J Mol Med. 2012;90:865-75.

12. D'Alessandra Y, Devanna P, Limana F, Straino S, Di Carlo A, Brambilla PG, et al. Circulating microRNAs are new and sensitive biomarkers of myocardial infarction. Eur Heart J. 2010;31:2765-73.

13. De Rosa S, Fichtlscherer S, Lehmann R, Assmus B, Dimmeler S, Zeiher AM. Transcoronary concentration gradients of circulating microRNAs. Circulation. 2011;124:1936-44. 
14. Devaux Y, Vausort M, McCann GP, Zangrando J, Kelly D, Razvi N, Zhang L, et al. MicroRNA-150: a novel marker of left ventricular remodeling after acute myocardial infarction. Circ Cardiovasc Genet. 2013;6:290-8.

15. Fichtlscherer S, De Rosa S, Fox H, Schwietz T, Fischer A, Liebetrau C, et al. Circulating microRNAs in patients with coronary artery disease. Circ Res. 2010;107:677-84.

16. Jaguszewski M, Osipova J, Ghadri JR, Napp LC, Widera C, Franke J, et al. A signature of circulating microRNAs differentiates takotsubo cardiomyopathy from acute myocardial infarction. Eur Heart J. 2014;35:999-1006.

17. van Rooij E, Sutherland LB, Thatcher JE, DiMaio JM, Naseem RH, Marshall WS, et al. Dysregulation of microRNAs after myocardial infarction reveals a role of miR-29 in cardiac fibrosis. Proc Natl Acad Sci U S A. 2008;105:13027-32.

18. Xiao J, Jing ZC, Ellinor PT, Liang D, Zhang H, Liu Y, et al. MicroRNA-134 as a potential plasma biomarker for the diagnosis of acute pulmonary embolism. J Transl Med. 2011;9:159.

19. Wang GK, Zhu JQ, Zhang JT, Li Q, Li Y, He J, et al. Circulating microRNA: a novel potential biomarker for early diagnosis of acute myocardial infarction in humans. Eur Heart J. 2010;31:659-66.

20. Catalucci D, Gallo P, Condorelli G. MicroRNAs in cardiovascular biology and heart disease. Circ Cardiovasc Genet. 2009;2:402-8.

21. Corsten MF, Dennert R, Jochems S, Kuznetsova T, Devaux Y, Hofstra L, et al. Circulating MicroRNA-208b and MicroRNA-499 reflect myocardial damage in cardiovascular disease. Circ Cardiovasc Genet. 2010;3:499-506.

22. Kuwabara Y, Ono K, Horie T, Nishi H, Nagao K, Kinoshita M, et al. Increased microRNA-1 and microRNA-133a levels in serum of patients with cardiovascular disease indicate myocardial damage. Circ Cardiovasc Genet. 2011:4:446-54.

23. Linsel-Nitschke $P$, Jansen $H$, Aherrarhou Z, Belz S, Mayer B, Lieb W, et al. Macrophage cholesterol efflux correlates with lipoprotein subclass distribution and risk of obstructive coronary artery disease in patients undergoing coronary angiography. Lipids Health Dis. 2009;8:14.

24. Mitchell PS, Parkin RK, Kroh EM, Fritz BR, Wyman SK, Pogosova-Agadjanyan EL, et al. Circulating microRNAs as stable blood-based markers for cancer detection. Proc Natl Acad Sci U S A. 2008;105:10513-8.

25. Fichtlscherer S, De Rosa S, Fox H, Schwietz T, Fischer A, Liebetrau C, et al. Circulating microRNAs in patients with coronary artery disease. Circ Res. 2010;107:677-84

26. Gentleman RC, Carey VJ, Bates DM, Bolstad B, Dettling M, Dudoit S, et al. Bioconductor: open software development for computational biology and bioinformatics. Genome Biol. 2004;5:R80
27. Livak KJ, Schmittgen TD. Analysis of relative gene expression data using real-time quantitative PCR and the 2(-Delta Delta C(T)) Method. Methods. 2001;25:402-8.

28. Smyth GK. Linear models and empirical bayes methods for assessing differential expression in microarray experiments. Stat Appl Genet Mol Biol. 2004;3:Article3

29. Benjamini Y, Hochberg Y. Controlling the False Discovery Rate: a Practical and Powerful Approach to Multiple Testing. R Stat Soc Series B Stat Methodol. 1995;57:289-300.

30. Ihaka R, Gentleman R. R: a language for data analysis and graphics. J Comp Graph Stat. 1996:5:299-314

31. Robin X, Turck N, Hainard A, Tiberti N, Lisacek F, Sanchez JC, et al. pROC: an open-source package for $\mathrm{R}$ and $\mathrm{S}+$ to analyze and compare ROC curves. BMC Bioinformatics. 2011;12:77.

32. Salaun PY, Couturaud F, Le Duc-Pennec A, Lacut K, Le Roux PY, Guillo P, et al. Noninvasive diagnosis of pulmonary embolism. Chest. 2011;139:1294-8

33. Torres-Macho J, Mancebo-Plaza AB, Crespo-Giménez A, Sanz de Barros MR, Bibiano-Guillén C, Fallos-Martí R, et al. Clinical features of patients inappropriately undiagnosed of pulmonary embolism. Am J Emerg Med. 2013;31:1646-50.

34. Smith SB, Geske JB, Maguire JM, Zane NA, Carter RE, Morgenthaler TI. Early anticoagulation is associated with reduced mortality for acute pulmonary embolism. Chest. 2010;137:1382-90.

35. Konstantinides S, Torbicki A. Management of venous thrombo-embolism: an update. Eur Heart J. 2014;35:2855-63.

36. Konstantinides S, Goldhaber SZ. Pulmonary embolism: risk assessment and management. Eur Heart J. 2012;33:3014-22.

37. van Es J, Douma RA, Gerdes VE, Kamphuisen PW, Buller HR. Acute pulmonary embolism. Part 2: treatment. Nat Rev Cardiol. 2010;7:613-22.

38. Amsterdam EA, Wenger NK, Brindis RG, Casey DE Jr, Ganiats TG, Holmes DR Jr, et al. 2014 AHA/ACC guideline for the management of patients with non-st-elevation acute coronary syndromes: a report of the American College of Cardiology/American Heart Association task force on practice guidelines. J Am Coll Cardiol. 2014;64:e139-228.

39. Stein PD, Sostman HD, Hull RD, Goodman LR, Leeper KV Jr, Gottschalk A, et al. Diagnosis of pulmonary embolism in the coronary care unit. Am J Cardiol. 2009:103:881-6.

40. Agewall S, Giannitsis E, Jernberg T, Katus H. Troponin elevation in coronary vs. non-coronary disease. Eur Heart J. 2011;32:404-11.

\section{Submit your next manuscript to BioMed Central and we will help you at every step:}

- We accept pre-submission inquiries

- Our selector tool helps you to find the most relevant journal

- We provide round the clock customer support

- Convenient online submission

- Thorough peer review

- Inclusion in PubMed and all major indexing services

- Maximum visibility for your research

Submit your manuscript at www.biomedcentral com/submit
BioMed Central 